Original article

https://www.journal-imab-bg.org

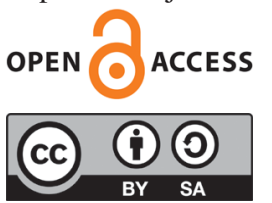

\title{
COMORBIDITY AND GOUT: ASSOCIATION BETWEEN HYPERURICEMIA AND BLOOD PARAMETERS AS A PREDICTOR OF GOUT
}

\author{
Erna Islamagic, Amra Lisovac, Muhamed Focak, Damir Suljevic \\ Department of Biochemistry and Physiology, Faculty of Science, University of \\ Sarajevo, Sarajevo, Bosnia and Herzegovina.
}

\section{SUMMARY}

Purpose: The aim of this study was to analyze the predictive potential of selected biochemical and hematological parameters in patients with gout from Bosnia and Herzegovina, and to determine the most common comorbidities related to gout.

Methods: Forty patients with gout (17 males and 23 females) from the hospital in the municipality of Novi Grad, Sarajevo were included in the present study. We analyzed biochemical parameters (uric acid, urea, creatinine, and CRP), ESR, and WBC as a hematological parameter. The Shapiro-Wilk test and the Spearman correlation coefficient were used for the normality and correlation analysis, respectively.

Results: Most gout patients were 50-70 years old and gout was more prevalent in female patients. Thirteen different comorbidities are related to gout. After the onset of the main disease, $82.35 \%$ of male patients were diagnosed with cardiovascular disease, while hypertension was diagnosed in $76.47 \%$ of male patients. High mean values of ESR, as well as CRP, uric acid, urea, and creatinine concentrations were found for all gout patients. A strong positive correlation was found after comparing urea and $\operatorname{ESR}(\rho=0.314)$; urea and creatinine $(\rho=0.526)$; CRP and $\operatorname{ESR}(\rho=0.375)$.

Conclusion: We can conclude that the analyzed biochemical parameters, CRP, urea, creatinine, and uric acid, as well as ESR, are good predictors of gout. High values of uric acid, creatinine, and urea are the most important gout predictors and significant markers for the development of comorbidities.

Keywords: gout, comorbidities, ESR, WBC, CRP, urea, creatinine, uric acid.

\section{INTRODUCTION}

Gout is a clinical syndrome that manifests as recurrent inflammation of one or more joints, resulting from the buildup of urate monohydrate crystals. Uric acid is the final degradation product in the purin metabolism and its concentration can be measured in both urine and serum [1]. Analysis of blood uric acid concentration helps to determine gout, with elevated values causing the formation of urate crystals that inflict damage on joints and other tissues, followed by development of inflammation [2]. Uric acid can be a marker for identification of systemic inflammation development [2]. Lower serum uric acid concentrations lead to a lower chance of development of acute gout attacks [3]. Uric acid values in female patients were about $20 \%$ lower than in male patients.

CRP is the most investigated inflammatory marker that promotes the association between cellular and humoral immunity, and is produced in the liver [4]. CRP values in gout are correlated with the number of affected joints. The CRP value starts to rise 4-6 hours after the inflammatory condition stimulus occurs, and the maximum value of this marker can be detected in the blood after 48-72 hours [5].

Electronegative charge on the membrane of red blood cells decreases under the conditions of increased acute phase protein in plasma. During acute inflammatory reaction such as gout, increased erythrocyte sedimentation rate occurs [6]. Red blood cell sedimentation value may increase up to three times, reaching the maximum seven days after the initial stimulus [2]. Leukocyte counts are often increased during elevated uric acid conditions, but without statistical significance [2].

During gout progression, kidney damage occurs and this process results in increased levels of creatinine and urea. Research shows that values of ceruloplasmin and complement proteins might also be increased by $50 \%$, while CRP and amyloid A serum proteins may be increased up to 1000 times during an inflammatory state such as gout $[4,7]$.

Gout is associated with many diseases, such as obesity, chronic kidney disease, hypertension, type 2 diabetes, dyslipidemia, cardiac diseases and peripheral arterial disease. Various cardiac diseases are independently associated with gout and, consequently, with an increased frequency of cardiovascular deaths [8]. Patients with gout have a higher chance of developing obesity than those without gout, according to Choi HK, et al. [9]. Links between hyperuricemia/gout and stroke have also been reported [10]. Research by Mikuls et al. (2005) showed that patients with gout are much more likely to have renal impairment than those with osteoarthritis [1]. There is a complex relationship between gout and diabetes. Authors observed that people with increased $\mathrm{HbA} 1 \mathrm{c}$ might be at risk of develop- 
ing hyperuricemia and gout [11]. These comorbidities are known to have a negative association with a lower quality of life [12]. Comorbidities and their treatment may have an effect on the development of gout [13].

The main aim of this research was to analyze the relationship between comorbidities and gout, with a particular focus on values of biochemical and hematological parameters in gout and on the predictive strength of those parameters.

\section{MATERIAL AND METHODS \\ Study design}

Forty patients with gout (17 males and 23 females) from the hospital of the municipality Novi Grad, Sarajevo were included in the present study. The research was performed in the period from May 2016 to May 2017. This study was approved by the Ethical Committee, Public Institution Health Center Sarajevo. Patients with a confirmed diagnosis of gout were included in the study. In addition to the main diagnosis, comorbidity was also included. Severely ill patients, as well as those with severe acute and chronic inflammation that can significantly alter target markers for gout, were not included. We performed an analysis of biochemical parameters, such as the concentration of uric acid, urea, creatinine, and $\mathrm{C}$ reactive protein $(\mathrm{CRP})$, and hematological parameters: white blood cell count (WBC) and erythrocyte sedimentation rate (ESR).

\section{Biochemical and hematologic analysis}

Biochemical analyses were performed using Olympus AU 640 biochemical analyzer. Concentration of uric acid was determined using the enzymatic method with uricase; Jaffe method was used for measurement of creatinine concentration, and enzymatic method with urease for urea concentration. Turbidimetric latex agglutination method was used for detection of $\mathrm{C}$ reactive protein concentration. Erythrocyte sedimentation rate was determined by Westergreen method, while a Diagon D-cell 60 hematologic analyzer was used to determine the WBC.

\section{Statistical analysis}

Results were analyzed using IBM SPSS Statistics ver. 20.0 (Chicago, USA). Data was presented in the form of mean value, standard deviation and range. The results were shown in a box and whisker plot and scatter plot. The Shapiro-Wilk test was used for normality analysis and the Spearman correlation coefficient for correlation analysis.

\section{RESULTS}

The present study included forty patients with diagnosed gout from Novi Grad Sarajevo. Figure 1 presents two groups of gout patients, categorized by sex (male and female) and further subcategorized by age (20-30, 30-40, 40-50, 50-60, 60-70, 70-80, 80-90).

Fig. 1. Overview of male and female gout patients by age

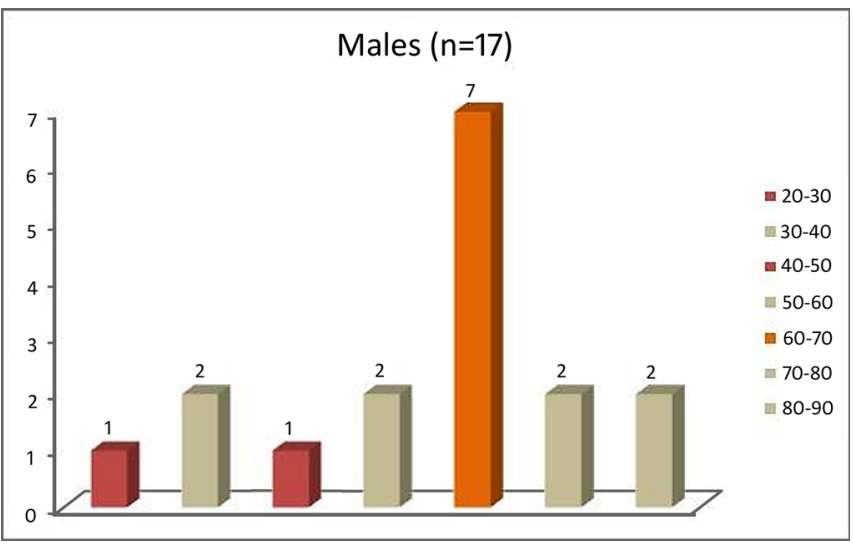

The study included more female patients with gout than male patients, even though the difference was not statistically significant. The highest number of gout patients was in the 50-70 age category. Gout was most common in male patients in the 60-70 age category and in female patients in the 50-60 age category. The lowest number of male patients was found in the 20-30 age category and the lowest number of female patients in the 8090 age category.

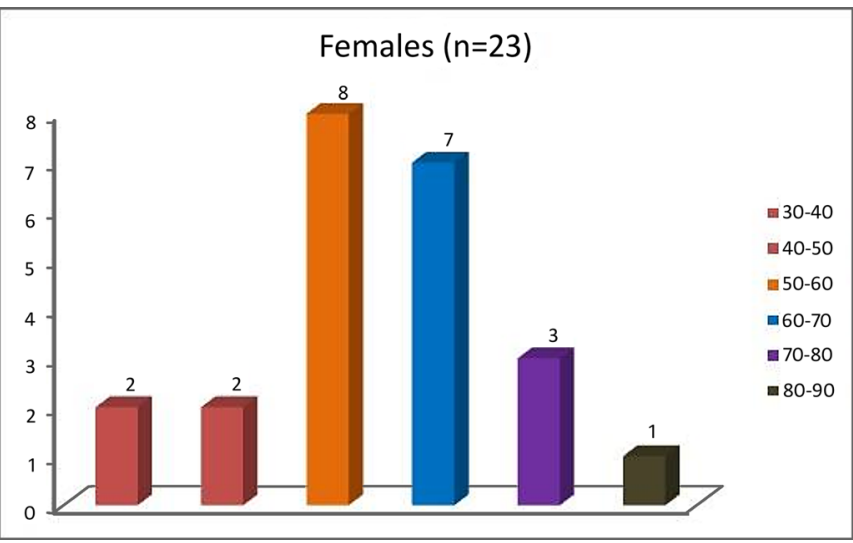

Figure 2 shows the incidence of comorbidity in patients with diagnosed gout. For male patients (A) there were 13 and for female patients (B) 11 comorbidities. Following the main disease, $82.35 \%$ of male patients were diagnosed with cardiovascular disease, while hypertension was diagnosed in $76.47 \%$ of male patients. For female patients, the presence of cardiovascular disease was $73.91 \%$, and hypertension $69.56 \%$. There was a significant incidence of hyperlipidemia and diabetes mellitus in several patients. 
Fig. 2. Frequency of comorbidity in male (A) and female (B) gout patients
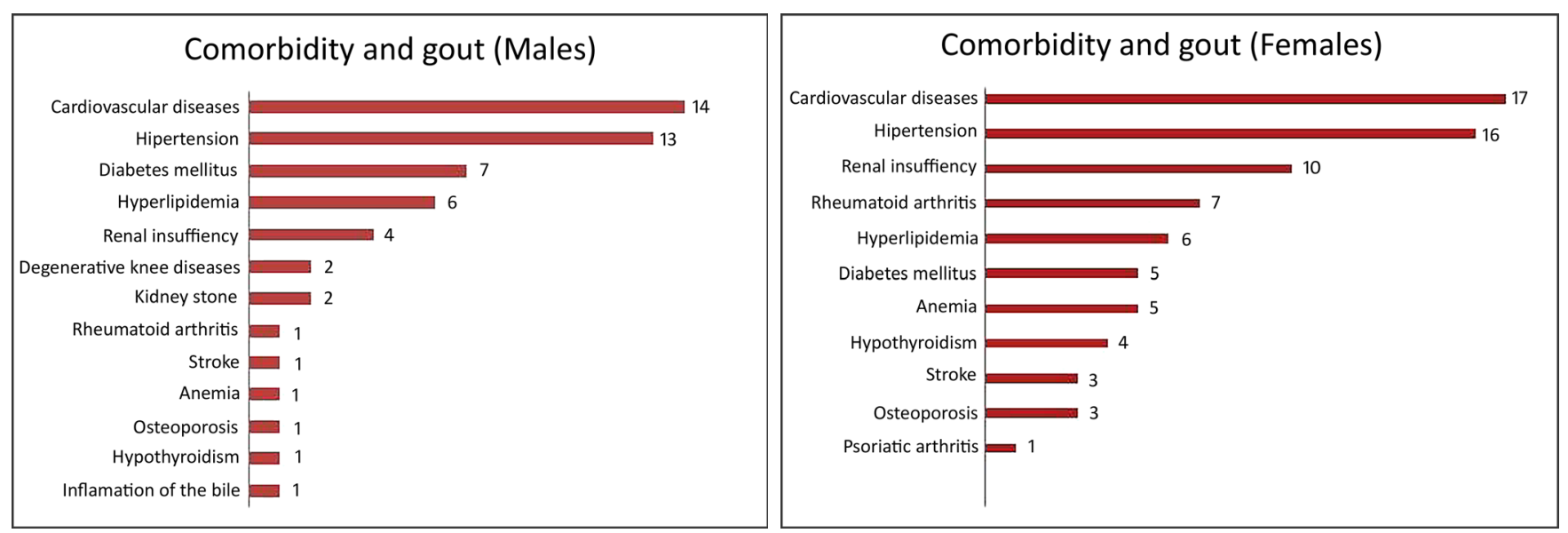

Table 1 presents mean value, standard deviation, range and Shapiro-Wilk normality test for biochemical parameters of patients with diagnosed gout.

Table 1. Values of biochemical parameters presented as mean \pm SD and range, and Shapiro-Wilk normality test

\begin{tabular}{|l|c|c|c|c|c|c|c|}
\hline \multicolumn{2}{|l|}{ Parameters } & $\begin{array}{c}\text { Uric acid } \\
(\mu \mathrm{mol} / \mathrm{L})\end{array}$ & $\begin{array}{c}\text { ESR } \\
(\mathrm{mm} / \mathrm{h})\end{array}$ & $\begin{array}{c}\text { CRP } \\
(\mathrm{mg} / \mathrm{L})\end{array}$ & $\begin{array}{c}\text { WBC } \\
\left(\mathrm{x} 10^{9} / \mathrm{L}\right)\end{array}$ & $\begin{array}{c}\text { Urea } \\
(\mathrm{mmol} / \mathrm{L})\end{array}$ & $\begin{array}{c}\text { Creatinine } \\
(\mu \mathrm{mol} / \mathrm{L})\end{array}$ \\
\hline \multirow{2}{*}{ Mean } & & $\begin{array}{c}508.13 \pm \\
109.12\end{array}$ & $\begin{array}{c}40.50 \pm \\
29.51\end{array}$ & $\begin{array}{c}20.72 \pm \\
21.59\end{array}$ & $9.18 \pm 3.40$ & $10.67 \pm 6.88$ & $\begin{array}{c}192.17 \pm \\
209.37\end{array}$ \\
\hline \multicolumn{2}{|l|}{ Range } & $364-817$ & $3-105$ & $0.60-93.7$ & $3.5-19.9$ & $2.3-29.6$ & $57-754$ \\
\hline \multirow{2}{*}{$\begin{array}{l}\text { Shapiro- } \\
\text { Wilk test }\end{array}$} & Stat. & .919 & .921 & .763 & .960 & .846 & .606. \\
\cline { 2 - 9 }$y$
\end{tabular}

* statistically significant at 0.05

Generally, for all patients, high mean values of ESR, as well as CRP, uric acid, urea, and creatinine concentrations were found, while WBC was in the reference range. However, the range of values had exceptionally high variations for all parameters in some patients, especially for uric acid, CRP, and creatinine. On the other hand, high standard deviations were found for all parameters. Except for WBC, the Shapiro-Wilk test showed a significant deviation of normality.
We compared the concentrations of biochemical parameters and values of hematologic parameters in male and female patients with gout (Figure 3). In general, higher mean values were found in males for WBC, CRP, uric acid, and creatinine, but statistically significant values $(p<0.05)$ were found only for uric acid. However, female patients had higher ESR values, but this was not statistically significant.

Fig. 3. Hematological (WBC, ESR) and biochemical parameters (CRP, uric acid, urea, creatinine) in male and female patients with gout. Data are presented in a box and whisker plot showing the upper and lower values (highest and lowest horizontal line, respectively) and upper and lower quartile (box) with median value. Male gout patients had markedly higher uric acid values $(\mathrm{p}<0.05)$ than female patients.
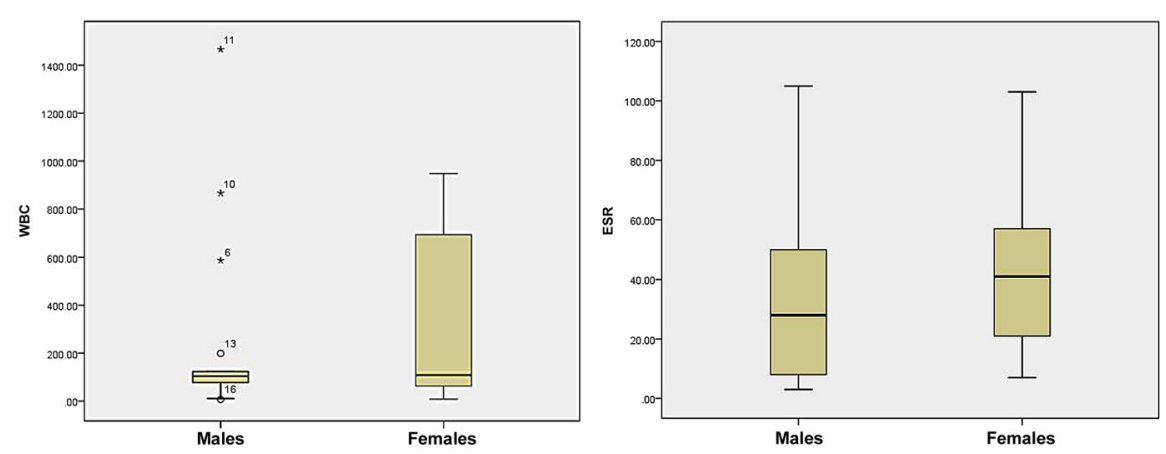

https://www.journal-imab-bg.org

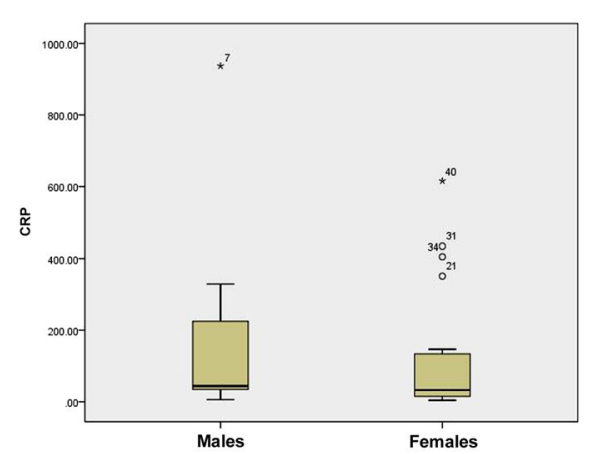

J of IMAB. 2020 Apr-Jun;26(2) 



We compared the parameters (WBC, ESR, CRP, uric acid, urea, and creatinine) in order to identify any positive or negative correlation between renal function markers and overall body function parameters (Figure 4). Spearman's rank correlation coefficient was used to measure the strength and correlation between variables. A positive correlation was found for all compared parameters

(urea $v s$ uric acid, urea $v s$ ESR, urea $v s$ creatinine, creatinine vs uric acid, CRP vs ESR). However, a strong positive correlation was found after comparison of urea and ESR $(\rho=0.314 ; p<0.05)$; urea and creatinine $(\rho=0.526$; $p<0.05)$; CRP and ESR $(\rho=0.375 ; p<0.05)$. Positive and statistically significant correlation data are visually presented in a scatter plot (Figure 4).

Fig. 4. Relationship between selected hematological and biochemical parameters in patients with gout. Spearman's rank correlation coefficient $(\rho)$ showed a positive correlation between the following parameters: urea and ESR, urea and creatinine, CRP and ESR. The figure presents parameters with a statistically significant $(\mathrm{p}<0.05)$ positive correlation.

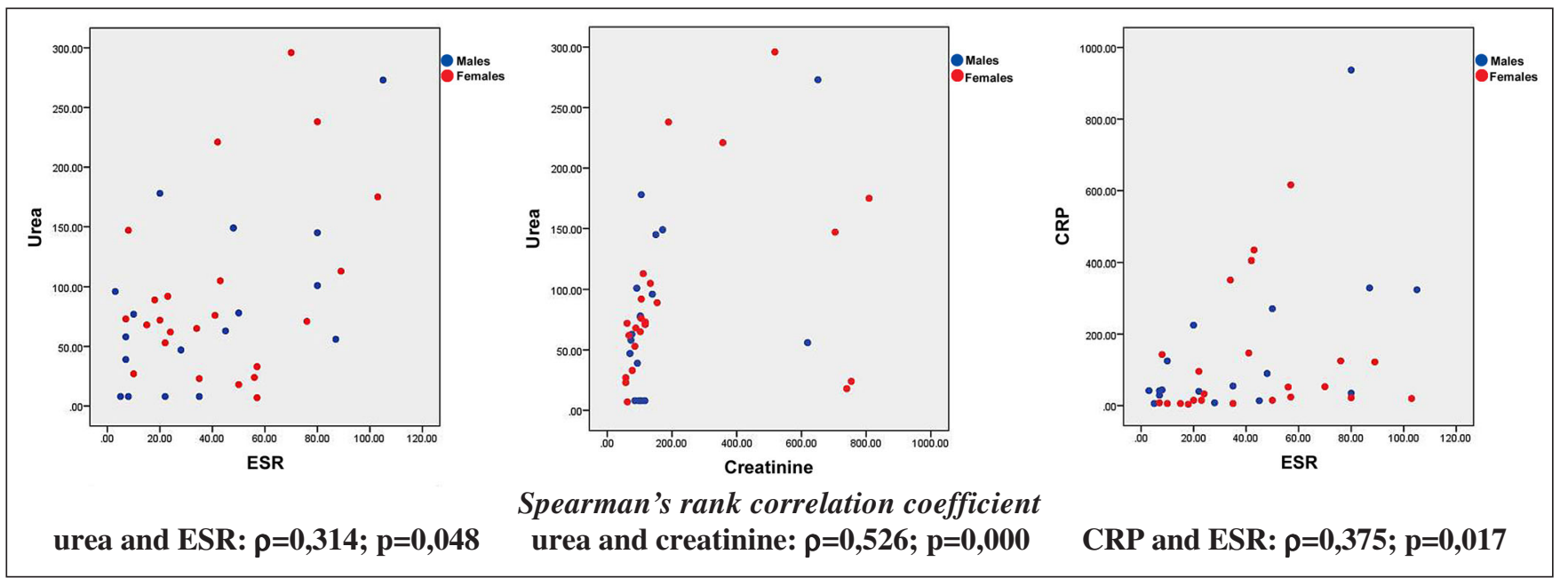

\section{DISCUSSION}

Gout is a disease that represents a global medical problem and the diagnosis is based on clinical signs, symptoms, and blood test results [14]. In the present study, we analyzed patients with gout categorized according to sex (males and females) and age (20-30; 30-40; 40-50; 50-60; 60-70; 70-80; 80-90 years).

Higher percentage of female patients than male patients with gout was observed in our study. The condition most commonly developed in male and female patients aged 50-60 years. Several studies considered gender differences in gout epidemiology and treatment, but they included a relatively small number of female patients with gout. In most studies, gout is predominantly labelled as a "male disease" and research was focused mainly on male patients. However, the incidence of gout in female and male patients over the age of 60 may be exactly the same; in females, the frequency of gout is significantly higher after menopause [15]. There is also a gender difference, considering that females during the reproductive period have lower values of serum uric acid. This is explained by the effects of estrogen: increased uric acid secretion and inhibition of renal urinary reabsorption via transporter for organic anions [15]. Janssens et al. (2010) also found a higher frequency of gout in male patients, which was not the case in our research [14]. The reason for this may be a small number of patients involved in our study.

In all analyzed gout patients, the values of urea, uric acid, creatinine, sedimentation and CPR were higher than the reference values. Regarding gender differences, it was found that significantly higher uric acid values were observed in males than in females. An increase in serum uric acid level has been established in many studies [8]. In our research, a positive correlation was found between urea and creatinine values, as well as between ESR and urea values. A positive correlation was also established between 
CRP and ESR values. As far as hematological parameters are concerned, mean values of WBC were in the reference range; however individual variations were found.

It is very important to emphasize the presence of comorbidity in patients with gout. In general, gout seems to correlate with cardiovascular disease $(82.35 \%$ for males, $73.91 \%$ for females), hypertension $(76.47 \%$ for males, $69.56 \%$ for females). Renal insufficiency in female patients with gout $(43.47 \%)$ was ranked third in frequency, while in male patients it was in the fifth place $(23.52 \%)$. Creatinine concentration values in female patients were significantly higher than in male patients, which is associated with a higher rate of renal disease such as renal insufficiency in female patients. Therefore, high creatinine values in gout patients, especially females, may be an important marker for renal insufficiency. The same number of males and females had hyperlipidemia in addition to the main disease. The highest risk was observed in the population of males and females aged 45-54 years. For patients under the age of 45 , this relationship was not confirmed [12]. Higher levels of uric acid are correlated with obesity, hypertension, hypertriglyceridemia, hypercholesterolemia, and lower level of high-density lipoproteins [12]. In patients with diabetes, a correlation between higher (> 80\%) uric acid concentrations and all-cause mortality has been observed [11].

Some recent research shows that concentrations of uric acid greater than $324 \mu \mathrm{mol} / \mathrm{L}$ in male and greater than $258 \mu \mathrm{mo} / \mathrm{L}$ in female patients are positively correlated (OR 5.1 for male and 5.0 for female) with other factors, such as coronary diseases. Some authors suggest an adverse effect of uric acid on endothelial function, oxidative metabolism and platelet aggregation, which can lead to endothelial dysfunction and blood vessel remodeling by oxidative stress $[16,17]$.

The relationship between hyperuricemia and metabolic syndrome had been already recognized. The presence of hyperuricemia was predisposed by the increasing frequency of risk factors, such as obesity and metabolic syndrome [18].
Authors showed that serum uric acid in postmenopause was significantly lower than in females without gout. Serum uric acid may be a protective factor of bone metabolism in primary osteoporosis patients [19]. Uric acid may have a protective role in bone metabolism, owing to its antioxidant effect $[20,21]$. A positive relationship between uric acid and bone health has been reinforced by a recently published meta-analysis by Veronese et al. (2016), including 55,859 participants [17]. Uric acids can suppress osteoclastogenesis and decrease the production of reactive oxygen species in osteoclast precursors [17].

Anemia is also identified as a risk factor for gout. In the study of McAdams-DeMarco et al., anemia was associated with an approximately two-fold increase in risk of developing gout [16,22]. In our study, five female patients and one male patient had gout and anemia. The biological mechanism linking anemia and uric acid level is still unknown and should be investigated in further studies.

\section{CONCLUSIONS}

Gout was more present in female patients, compared to male patients. Regarding comorbidities, cardiovascular disease, hypertension, hyperlipidemia and diabetes mellitus were the most common in male and female patients with gout. High mean values of ESR and other analyzed biochemical parameters were found for both male and female gout patients, while WBC was in the reference range. A strong positive correlation was found when we compared urea $v s$ ESR, urea $v s$ creatinine and CRP $v s$ ESR. There was a strong association between gout and comorbidity, such as cardiovascular disease, hypertension, hyperlipidemia and renal disease.

Hyperuricemia associated with hypertension can cause cardiovascular disease, and the incidence increases with lower glomerular filtrations followed with high creatinine values. Therefore, hyperuricemia with high creatinine and urea value is the most important gout predictor and a significant marker for the development of gout-related comorbidity.

\section{REFERENCES:}

1. Mikuls T, Farrar J, Bilker W, Fernandes S, Schumacher HR Jr, Saag KG. Gout epidemiology: results from the UK general practice research database, 1990-1999. Ann Rheum Dis. 2005 Feb;64:267-72. [PubMed] [Crossref]

2. Ruggiero C, Cherubini A, Ble A, Bos A, Maggio M, Dixit VD, et al. Uric acid and inflammatory markers. Eur Heart J. 2006 May;27(10):1174-81. [PubMed] [Crossref]

3. Xiang LW, Li J, Lin JM, Li HF. Determination of gouty arthritis' biomarkers in human urine using re- versed-phase high-performance liquid chromatography. J Pharm Anal. 2014 Apr;4(2):153-8. [PubMed] [Crossref]

4. Rosa Neto NS, de Carvalho JF. [The use of inflammatory laboratory tests in rheumatology.] [In Portuguese] Rev Bras Reumatol. 2009 July-Aug; 49(4). [Crossref]

5. Tagoe CE, Raza Y. Differences in Acute Phase Reactants between Gout and Pseudogout. Int J Clin Med. 2013 Jan;4:13-9. [Crossref]

6. Lee JH, Yang JA, Shin K, Lee GH, Lee WW, Lee EY, et al. Elderly Patients Exhibit Stronger Inflammatory
Responses during Gout Attacks. J Korean Med Sci. 2017 Dec;32(12):196773. [PubMed] [Crossref]

7. ter Borg E, Wegewijs MA, de Bruin P. Gout and AA Amyloidosis: A Case Report and Review of the Literature. J Clin Rheumatol. 2017 Jun; 23(4):233-4. [PubMed] [Crossref]

8. Clarson LE, Hider SL, Belcher J, Heneghan C, Roddy E, Mallen CD. Increased risk of vascular disease associated with gout: a retrospective, matched cohort study in the UK clinical practice research datalink. Ann Rheum Dis. 2015 Apr;74(4):642-7. 


\section{[PubMed] [Crossref]}

9. Choi HK, Ford ES, Li C, Curhan

G. Prevalence of the metabolic syndrome in patients with gout: the Third National Health and Nutrition Examination Survey. Arthritis Rheum. 2007 Feb;57(1):109-15. [PubMed] [Crossref]

10. Kim S, Guevara J, Kim K, Choi HK, Heitjan DF, Albert DA. Hyperuricemia and risk of stroke: a systematic review and meta-analysis. Arthritis Care Res. 2009 Jul;61:88592. [PubMed] [Crossref]

11. Choi HK, Ford ES. Haemoglobin A1c, fasting glucose, serum Cpeptide and insulin resistance in relation to serum uric acid levels-the Third National Health and Nutrition Examination Survey. Rheumatology. 2008 May;47:713-7. [PubMed] [Crossref]

12. Singh JA. The impact of gout on patient's lives: a study of AfricanAmerican and Caucasian men and women with gout. Arthritis Res Ther. 2014 Jun;16(3):R132. [PubMed] [Crossref]

13. Stamp LK, Chapman PT. Gout and its comorbidities: implications for therapy. Rheumatology (Oxford). 2013 Jan; 52(1): 34-44. [PubMed] [Crossref]

14. Janssens HJ, Fransen J, van de
Lisdonk EH, van Riel PL, van Weel C, Janssen M. A diagnostic rule for acute gouty arthritis in primary care without joint fluid analysis. Arch Intern Med. 2010 Jul;170(13):1120-6. [PubMed] [Crossref]

15. Bernal JA, Quilis N, Andrés M, Sivera F, Pascual E. Gout: optimizing treatment to achieve a disease cure. Ther Adv Chronic Dis. 2016 Mar;7(2):135-44. [PubMed] [Crossref]

16. McAdams-DeMarco MA, Maynard JW, Coresh J, Baer AN. Anemia and the onset of gout in a population-based cohort of adults: Atherosclerosis Risk in Communities study. Arthritis Res Ther. 2012 Aug; 14(4):R193. [PubMed] [Crossref]

17. Veronese N, Carraro S, Bano G, Trevisan C, Solmi M, Luchini C, et al. Hyperuricemia protects against low bone mineral density, osteoporosis and fractures: a systematic review and meta-analysis. Eur J Clin Invest. 2016 Nov;46(11):920-30. [PubMed] [Crossref]

18. Zhu Y, Pandya BJ, Choi HK. Prevalence of gout and hyperuricemia in the US general population: the $\mathrm{Na}$ tional Health and Nutrition Examina- tion Survey 2007-2008. Arthritis Rheum. 2011 Oct; 63(10): 3136-41. [PubMed] [Crossref]

19. Chen L, Peng Y, Fang F, Chen J, Pan L, You L. Correlation of serum uric acid with bone mineral density and fragility fracture in patients with primary osteoporosis: a single-center retrospective study of 253 cases. Int J Clin Exp Med. 2015 Apr; 8(4):6291-4. [PubMed]

20. Kaushal N, Vohora D, Jalali RK, Jha $\mathrm{S}$. Raised serum uric acid is associated with higher bone mineral density in a cross-sectional study of a healthy Indian population. Ther Clin Risk Manag. 2018 Jan;14:75-82. [PubMed] [Crossref]

21. Lin KM, Lu CL, Hung KC, Wu PC, Pan CF, Wu CJ, et al. The Paradoxical Role of Uric Acid in Osteoporosis. Nutrients. 2019 Sep;11(9): 2111. [PubMed] [Crossref]

22. Eun Y, Han KD, Kim DH, Kim IY, Park EJ, Lee S, et al. Association between anemia and hyperuricemia: results from the Korean National Health and Nutrition Examination Survey. Sci Rep. 2019 Dec;9(1):19067. [PubMed] [Crossref]

Please cite this article as: Islamagic E, Lisovac A, Focak M, Suljevic D. Comorbidity and gout: association between hyperuricemia and blood parameters as a predictor of gout. J of IMAB. 2020 Apr-Jun;26(2):3092-3097.

DOI: https://doi.org/10.5272/jimab.2020262.3092

Received: 22/05/2019; Published online: 24/04/2020



Address for correspondence

Erna Islamagic, MA

Faculty of Science, University of Sarajevo

Zmaja od Bosne 33-35, 71000 Sarajevo, Bosnia and Herzegovina

E-mail: erna.islamagic@hotmail.com, 